\title{
Some Approximation Properties of Series with Nonlinear Fourier Basis
}

\author{
Hatice Aslan* and Ali Guven
}

(Communicated by Nihal YILMAZ ÖZGÜR)

\begin{abstract}
The order of approximation of generalized de la Vallée Poussin means of series with nonlinear Fourier basis was investigated in uniform and Hölder norms.
\end{abstract}

Keywords: Generalized de la Vallée Poussin mean; nonlinear Fourier basis; order of approximation.

AMS Subject Classification (2010): 41A25; $42 A 10$.

*Corresponding Author

\section{Introduction and main results}

The order of approximation by means of classical Fourier series (Fourier series with linear basis) were investigated by several authors. Results of these studies can be found in the excellent monographs [1], [10], [11], and in the survey [2]. The order of approximation by generalized de la Vallée Poussin means of classical Fourier series of $2 \pi$-periodic functions was firstly investigated by L. Leindler in the uniform norm ([4]). Later, Z. Stypinski ([9]) obtained an estimate for these means in the Hölder norm. The aim of this article is to obtain estimates for the approximation order of generalized de la Vallée Poussin means of series with nonlinear Fourier basis in uniform and Hölder norms.

Let $\mathbb{D}=\{z \in \mathbb{C}:|z|<1\}$ and $a \in \mathbb{D}$. We consider the Möbius transformation

$$
\tau_{a}(z)=\frac{z-a}{1-\bar{a} z}
$$

which is a conformal automorphism of $\mathbb{D}$. The nonlinear phase function $\theta_{a}$ is defined through the relation

$$
e^{i \theta_{a}(t)}:=\tau_{a}\left(e^{i t}\right)=\frac{e^{i t}-a}{1-\bar{a} e^{i t}},
$$

where $\tau_{a}\left(e^{i t}\right)$ stands for radial boundary value of $\tau_{a}$. It is easy to see that $\theta_{a}(t+2 \pi)=\theta_{a}(t)+2 \pi$, and if we set $a=|a| e^{i t_{a}}$, then

which satisfies

$$
\theta_{a}^{\prime}(t)=p_{a}(t):=\frac{1-|a|^{2}}{1-2|a| \cos \left(t-t_{a}\right)+|a|^{2}},
$$

$$
\frac{1-|a|}{1+|a|} \leq p_{a}(t) \leq \frac{1+|a|}{1-|a|} .
$$

Let $\mathbb{T}=\mathbb{R} / 2 \pi \mathbb{Z}$. The space $L_{a}^{2}(\mathbb{T})$, consists of measurable functions $f: \mathbb{T} \rightarrow \mathbb{C}$ such that

$$
\frac{1}{2 \pi} \int_{\mathbb{T}}|f(t)|^{2} p_{a}(t) d t<\infty
$$

Received : 06-06-2018, Accepted : 13-09-2018 
becomes a Hilbert space with respect to the inner product

$$
\langle f, g\rangle_{a}:=\frac{1}{2 \pi} \int_{\mathbb{T}} f(x) \overline{g(x)} p_{a}(x) d x,
$$

and the set

$$
\left\{e^{i k \theta_{a}(x)}: k \in \mathbb{Z}\right\}
$$

is an orthonormal basis for $L_{a}^{2}(\mathbb{T})$ (see [7] and [8]). In the case $a=0$ we obtain the classical Fourier basis $\left\{e^{i k x}: k \in \mathbb{Z}\right\}$ for the space $L_{0}^{2}(\mathbb{T})=L^{2}(\mathbb{T})$.

Fourier series of a function $f \in L^{1}(\mathbb{T})$ with respect to the orthonormal basis $\left\{e^{i k \theta_{a}(x)}: k \in \mathbb{Z}\right\}$ become

$$
f(x) \sim \sum_{k=-\infty}^{\infty} c_{k}^{a}(f) e^{i k \theta_{a}(x)}
$$

where

$$
c_{k}^{a}(f)=\frac{1}{2 \pi} \int_{\mathbb{T}} f(x) e^{i k \theta_{a}(x)} p_{a}(x) d x, k \in \mathbb{Z} .
$$

If we denote the $n$th partial sum of this series by $S_{n}^{a}(f)(x)$, then we have

$$
\begin{aligned}
S_{n}^{a}(f)(x) & =\frac{1}{2 \pi} \int_{\mathbb{T}} f(t) D_{n}\left(\theta_{a}(x)-\theta_{a}(t)\right) p_{a}(t) d t \\
& =\frac{1}{2 \pi} \int_{\mathbb{T}} F\left(\theta_{a}(x)+t\right) D_{n}(t) d t
\end{aligned}
$$

where $F:=f \circ \theta_{a}^{-1}$ and $D_{n}$ is the Dirichlet kernel of order $n$.

We denote by $C(\mathbb{T})$ the Banach space of continuous functions $f: \mathbb{R} \rightarrow \mathbb{C}$ equipped with the norm

$$
\|f\|_{\infty}:=\sup _{x \in \mathbb{T}}|f(x)| .
$$

The modulus of continuity of $f \in C(\mathbb{T})$ is defined by

$$
\omega(f, t):=\sup _{0<h \leq t}\|f(\cdot+h)-f\|_{\infty}
$$

for $t>0$.

For $0<\alpha \leq 1$ the Hölder space $H^{\alpha}(\mathbb{T})$ is the space of functions $f \in C(\mathbb{T})$ such that

$$
\omega(f, t) \lesssim t^{\alpha}, t>0
$$

or equivalently

$$
\Delta^{\alpha}(f):=\sup _{x \neq y} \frac{|f(x)-f(y)|}{|x-y|^{\alpha}}<\infty .
$$

$H^{\alpha}(\mathbb{T})$ becomes a Banach space with respect to the Hölder norm

$$
\|f\|_{\alpha}:=\|f\|_{\infty}+\Delta^{\alpha}(f) .
$$

For each natural number $n$, let $\Pi_{n}^{a}$ be the set of all nonlinear trigonometric polynomials of degree at most $n$, that is

$$
\Pi_{n}^{a}:=\operatorname{span}\left\{e^{i k \theta_{a}(x)}:|k| \leq n\right\},
$$

and let $E_{n}^{a}(f)$ be the approximation error of $f \in C(\mathbb{T})$ by elements of $\Pi_{n}^{a}$, i. e.

$$
E_{n}^{a}(f):=\inf _{T \in \Pi_{n}^{a}}\|f-T\|_{\infty} .
$$


Let $\lambda=\left\{\lambda_{n}\right\}$ be a sequence of integers such that $\lambda_{1}=1$ and $0 \leq \lambda_{n+1}-\lambda_{n} \leq 1$. The sequence of generalized de la Vallée Poussin means of the series 1.1 is defined by

$$
V_{n}^{a}(\lambda, f)=\frac{1}{\lambda_{n}} \sum_{k=n-\lambda_{n}}^{n-1} S_{k}^{a}(f) .
$$

In special case $\lambda_{n}=1(n=1,2, \ldots) V_{n}^{a}(\lambda, f)$ become $S_{n-1}^{a}(f)$ and in the case $\lambda_{n}=n(n=1,2, \ldots)$ we have the Fejér means

$$
V_{n}^{a}(\lambda, f)=\sigma_{n-1}^{a}(f):=\frac{1}{n} \sum_{k=0}^{n-1} S_{k}^{a}(f) .
$$

In the rest of the article, $A \lesssim B$ will mean that there exists an absolute constant $c>0$ such that $A \leq c B$ holds for quantities $A$ and $B$.

Our main results are the following.

Theorem 1.1. Let $\lambda=\left\{\lambda_{n}\right\}$ be a sequence of integers such that $\lambda_{1}=1$ and $0 \leq \lambda_{n+1}-\lambda_{n} \leq 1$. If $f \in C$ (T) satisfies $|f(x)| \leq M$, then the estimate

$$
\left\|V_{n}^{a}(\lambda, f)\right\|_{\infty} \leq M\left(3+\log \frac{2 n-\lambda_{n}}{\lambda_{n}}\right)
$$

holds for every natural number $n$.

Theorem 1.2. For $f \in C(\mathbb{T})$, the degree of approximation by the sequence $\left\{V_{n}^{a}(\lambda, f)\right\}$ of generalized de la Vallée Poussin means is estimated as

$$
\left\|f-V_{n}^{a}(\lambda, f)\right\|_{\infty} \lesssim\left(3+\log \frac{2 n-\lambda_{n}}{\lambda_{n}}\right) E_{n-\lambda_{n}}^{a}(f) .
$$

From this estimate and Theorem 2.3 of [3] we get the following analogue of Lebesgue's inequality.

Corollary 1.1. If $f \in H^{\alpha}(\mathbb{T})(0<\alpha \leq 1)$, then

$$
\left\|f-S_{n}^{a}(f)\right\|_{\infty} \lesssim\left(\frac{1+|a|}{1-|a|}\right)^{\alpha} \frac{\log n}{n^{\alpha}} .
$$

Theorem 1.3. Let $\lambda=\left\{\lambda_{n}\right\}$ be a sequence of integers such that $\lambda_{1}=1$ and $0 \leq \lambda_{n+1}-\lambda_{n} \leq 1$. If $f \in H^{\alpha}(\mathbb{T})(0<\alpha \leq 1)$ then

$$
\left\|f-V_{n}^{a}(\lambda, f)\right\|_{\infty} \lesssim\left\{\begin{array}{cc}
\left(\frac{1+|a|}{1-|a|}\right)^{\alpha} \frac{1}{\lambda_{n}^{\alpha}}, & \alpha<1 \\
\left(\frac{1+|a|}{1-|a|}\right) \frac{1+\log \lambda_{n}}{\lambda_{n}}, & \alpha=1 .
\end{array}\right.
$$

Theorem 1.4. Let $\lambda=\left\{\lambda_{n}\right\}$ be a sequence of integers such that $\lambda_{1}=1$ and $0 \leq \lambda_{n+1}-\lambda_{n} \leq 1,0 \leq \beta<\alpha \leq 1$ and let $f \in H^{\alpha}(\mathbb{T})$. Then

$$
\left\|f-V_{n}^{a}(\lambda, f)\right\|_{\beta} \lesssim\left\{\begin{array}{cc}
\left(\frac{1+|a|}{1-|a|}\right)^{\alpha} n^{\beta-\alpha} \frac{1}{\lambda_{n}^{\alpha}} \log \frac{2 n}{\lambda_{n}}, & \alpha<1 \\
\left(\frac{1+|a|}{1-|a|}\right)^{\beta-1} \frac{1+\log \lambda_{n}}{\lambda_{n}} \log \frac{2 n}{\lambda_{n}}, & \alpha=1 .
\end{array}\right.
$$

Corollary 1.2. If $0 \leq \beta<\alpha \leq 1$ and $f \in H^{\alpha}(\mathbb{T})$, then

$$
\left\|f-\sigma_{n}^{a}(f)\right\|_{\beta} \lesssim\left\{\begin{array}{cc}
\left(\frac{1+|a|}{1-|a|}\right)^{\alpha} \frac{1}{n^{2 \alpha-\beta}}, & \alpha<1 \\
\left(\frac{1+|a|}{1-|a|}\right) \frac{\log n}{n^{2-\beta}}, & \alpha=1 .
\end{array}\right.
$$

\section{Auxiliary Results}

Lemma 2.1. [4] Let $g \in L^{1}\left(0, \frac{\pi}{2}\right)$ and $|g(t)| \leq M$ for $t \in(0, \delta)\left(\delta \leq \frac{\pi}{2}\right)$. If we set

$$
I\left(g, \lambda_{n}\right):=\int_{0}^{\frac{\pi}{2}} g(t) \frac{\left|\sin \lambda_{n} t \sin \left(2 n-\lambda_{n}\right) t\right|}{\lambda_{n} \sin ^{2} t} d t
$$


then we have

$$
I\left(g, \lambda_{n}\right) \leq M\left(3+\log \frac{2 n-\lambda_{n}}{\lambda_{n}}\right)+\frac{1}{\lambda_{n}} J(g, \delta),
$$

where $J(g, \delta)$ is independent of $\lambda_{n}$ and $J\left(g, \frac{\pi}{2}\right)=0$.

Let $\varphi$ be an increasing positive function on $(0, \infty)$. The $\varphi$-norm of a function $f \in C(\mathbb{T})$ is defined by

$$
\|f\|_{\varphi}:=\|f\|_{\infty}+\sup _{x \neq y} \frac{|f(x)-f(y)|}{\varphi(|x-y|)}=\|f\|_{\infty}+\sup _{\delta>0} \frac{\|f-f(\cdot+\delta)\|_{\infty}}{\varphi(\delta)} .
$$

It is clear that, in the spacial case $\varphi(\delta)=\delta^{\alpha}(0<\alpha \leq 1)$ we have $\|f\|_{\varphi}=\|f\|_{\alpha}$.

The following important result was obtained in [5].

Lemma 2.2. Let $\left\{A_{n}\right\}$ be a sequence of linear convolution operators from $C(\mathbb{T})$ into $C(\mathbb{T})$, and let $\varphi$ be an increasing positive function on $(0, \infty)$. Then

$$
\left\|A_{n}(f)-f\right\|_{\varphi} \leq\left\|A_{n}(f)-f\right\|_{\infty}\left(1+\frac{2}{\varphi(1 / n)}\right)+\sup _{0<\delta \leq 1 / n} \frac{2 \omega(f, \delta)}{\varphi(\delta)}\left(1+\left\|A_{n}\right\|\right)
$$

holds for every $f \in C(\mathbb{T})$, where $\left\|A_{n}\right\|$ is the operator norm of $A_{n}$.

\section{Proofs of Main Results}

Proof of Theorem 1.1. From (1.2), a standard computation gives that

$$
V_{n}^{a}(\lambda, f)(x)=\frac{1}{\lambda_{n}} \sum_{k=n-\lambda_{n}}^{n-1} \frac{1}{2 \pi} \int_{0}^{\pi}\left\{F\left(\theta_{a}(x)+t\right)-F\left(\theta_{a}(x)-t\right)\right\} D_{k}(t) d t,
$$

where $D_{k}(t)$ Dirichlet kernel. By using Dirichlet kernel's property (see in [11]).

$$
\begin{aligned}
V_{n}^{a}(\lambda, f)(x) & =\frac{1}{2 \pi \lambda_{n}} \int_{0}^{\pi}\left\{F\left(\theta_{a}(x)+t\right)-F\left(\theta_{a}(x)-t\right)\right\}\left(\sum_{k=n-\lambda_{n}}^{n-1} D_{k}(t)\right) d t \\
& =\frac{1}{\pi \lambda_{n}} \int_{0}^{\pi}\left\{F\left(\theta_{a}(x)+t\right)-F\left(\theta_{a}(x)-t\right)\right\} \frac{\sin \lambda_{n} t \sin \left(2 n-\lambda_{n}\right) t}{\sin ^{2} t} d t .
\end{aligned}
$$

By changing variables we obtain the following inequality.

$$
V_{n}^{a}(\lambda, f)(x)=\frac{1}{\pi \lambda_{n}} \int_{0}^{\frac{\pi}{2}}\left\{F\left(\theta_{a}(x)+2 t\right)-F\left(\theta_{a}(x)-2 t\right)\right\} \frac{\sin \lambda_{n} t \sin \left(2 n-\lambda_{n}\right) t}{\sin ^{2} t} d t .
$$

Applying Lemma 2.1 with $g(t)=F\left(\theta_{a}(x)+2 t\right)-F\left(\theta_{a}(x)-2 t\right)$ and $\delta=\frac{\pi}{2}$, we get inequality (1.3) .

Proof of Theorem 1.2. Let $T^{*}(x)$ denote the trigonometric polynomial of best approximation of order not bigger than $m$. From the definitions of $S_{n}^{a}(f)$ and $V_{n}^{a}(f)$ it is clear that if $n-\lambda_{n} \geqq m$, then

$$
V_{n}^{a}\left(f-T_{m}^{*}\right)(x) \lesssim V_{n}^{a}(f)(x)-T_{m}^{*}(x) .
$$

Hence we have

$$
\begin{aligned}
V_{n}^{a}\left(f-T_{m}^{*}\right)(x) & \lesssim\left|V_{n}^{a}(f)(x)-T_{n-\lambda_{n}}^{*}(x)\right|+\left|T_{n-\lambda_{n}}^{*}(x)-f(x)\right| \\
& \lesssim\left|V_{n}^{a}\left(f-T_{n-\lambda_{n}}^{*}\right)(x)\right|+E_{n-\lambda_{n}}^{a}(f) .
\end{aligned}
$$

According to (3.1) we get

$$
\left|V_{n}^{a}\left(f-T_{n-\lambda_{n}}^{*}\right)(x)\right| \lesssim \frac{1}{\pi \lambda_{n}} \int_{0}^{\frac{\pi}{2}} 2 E_{n-\lambda_{n}}^{a}(f) \frac{\sin \lambda_{n} t \sin \left(2 n-\lambda_{n}\right) t}{\sin ^{2} t} d t .
$$


Using Lemma 2.1 with $g(t)=2 E_{n-\lambda_{n}}^{a}(f)$ and $\delta=\frac{\pi}{2}$, we obtain

$$
\left|V_{n}^{a}\left(f-T_{n-\lambda_{n}}^{*}\right)(x)\right| \lesssim\left(3+\log \frac{2 n-\lambda_{n}}{\lambda_{n}}\right) E_{n-\lambda_{n}}^{a}(f) .
$$

Proof of Theorem 1.3. Let

$$
\phi_{x}^{a}(t)=F\left(\theta_{a}(x)+2 t\right)-F\left(\theta_{a}(x)-2 t\right)-2 f(x) .
$$

Therefore since $f \in H^{\alpha}(\mathbb{T})$ and using the following inequality

$$
\left|\theta_{a}^{-1}(x)-\theta_{a}^{-1}(y)\right|=\left|\left(\theta_{a}^{-1}\right)^{\prime}(\xi)\right||x-y| \leq \frac{1}{\theta_{a}^{\prime}\left(\theta_{a}^{-1}(\xi)\right)}|x-y| \leq\left(\frac{1+|a|}{1-|a|}\right)|x-y|
$$

where $\xi \in(x, y)$ or $\xi \in(x, y)$, we have

$$
\phi_{x}^{a}(t) \leq M\left(\frac{1+|a|}{1-|a|}\right)^{\alpha} t^{\alpha} .
$$

If $f \in H^{\alpha}(\mathbb{T})$, we obtain by using $(3.2)$

$$
\begin{aligned}
\left|V_{n}^{a}(f)(x)-f(x)\right| & =\frac{1}{\pi \lambda_{n}} \int_{0}^{\frac{\pi}{2}}\left|\phi_{x}^{a}(t)\right| \frac{\sin \lambda_{n} t \sin \left(2 n-\lambda_{n}\right) t}{\sin ^{2} t} d t \\
& \leq \frac{1}{\pi \lambda_{n}} \int_{0}^{\frac{\pi}{2}} \phi_{x}^{a}(t) \frac{\left|\sin \lambda_{n} t \sin \left(2 n-\lambda_{n}\right) t\right|}{\sin ^{2} t} d t \\
& \lesssim\left(\frac{1+|a|}{1-|a|}\right)^{\alpha} \frac{1}{\pi \lambda_{n}} \int_{0}^{\frac{\pi}{2}} t^{\alpha} \frac{\left|\sin \lambda_{n} t \sin \left(2 n-\lambda_{n}\right) t\right|}{\sin ^{2} t} d t .
\end{aligned}
$$

Let us split the integral in three parts:

$$
\int_{0}^{\frac{\pi}{2}}=\int_{0}^{\frac{1}{2 n-\lambda_{n}}}+\int_{\frac{1}{2 n-\lambda_{n}}}^{\frac{1}{\lambda_{n}}}+\int_{\frac{1}{\lambda_{n}}}^{\frac{\pi}{2}} \equiv I_{1}+I_{2}+I_{3} .
$$

$I_{1}$ and $I_{2}$ can easily be estimated for any $\alpha \leqq 1$ as follows.

$$
\begin{aligned}
\frac{1}{\lambda_{n}} I_{1} & \lesssim \frac{1}{\lambda_{n}} \int_{0}^{\frac{1}{2 n-\lambda_{n}}} t^{\alpha} \lambda_{n}\left(2 n-\lambda_{n}\right) d t \lesssim \frac{1}{\lambda_{n}^{\alpha}} \\
\frac{1}{\lambda_{n}} I_{2} & \lesssim \frac{1}{\lambda_{n}} \int_{\frac{1}{2 n-\lambda_{n}}}^{\frac{1}{\lambda_{n}}} t^{\alpha-1} \lambda_{n} d t \lesssim \frac{1}{\alpha} \frac{1}{\lambda_{n}^{\alpha}} .
\end{aligned}
$$

The estimate of $I_{3}$ differs according to whether $\alpha<1$ or $\alpha=1$ :

$$
\frac{1}{\lambda_{n}} I_{3} \lesssim \frac{1}{\lambda_{n}} \int_{\frac{1}{\lambda_{n}}}^{\frac{\pi}{2}} t^{\alpha-2} d t \lesssim\left\{\begin{array}{cc}
\left(\frac{1}{1-\alpha}\right) \frac{1}{\lambda_{n}^{\alpha}}, & \alpha<1 \\
\frac{1+\log \lambda_{n}}{\lambda_{n}}, & \alpha=1 .
\end{array}\right.
$$

Collecting our estimates, we obtain (1.5) .

Proof of Theorem 1.4. Let $\varphi(\delta)=\delta^{\beta},\|f\|_{\varphi}=\|f\|_{\beta}, f \in H^{\alpha}(\mathbb{T}), 0 \leq \beta<\alpha \leq 1$. If $A_{n}=V_{n}^{a}$, then (2.2) yields

$$
\left\|V_{n}^{a}(f)-f\right\|_{\beta} \leq\left\|V_{n}^{a}(f)-f\right\|_{\infty}\left(1+\frac{2}{1 / n^{\beta}}\right)+\sup _{0<\delta \leq 1 / n} \frac{2 \omega(f, \delta)}{\delta^{\beta}}\left(1+\left\|V_{n}^{a}\right\|\right) .
$$

For $\alpha<1$, from Theorem 1.1 and Theorem 1.3, we have

$$
\begin{aligned}
\left\|V_{n}^{a}(\lambda, f)-f\right\|_{\beta} \lesssim & \left(\frac{1+|a|}{1-|a|}\right)^{\alpha} \frac{1}{\lambda_{n}^{\alpha}}\left(1+2 n^{\beta}\right) \\
& +\sup _{0<\delta \leq 1 / n} 2 \delta^{\alpha-\beta}\left(\frac{1+|a|}{1-|a|}\right)^{\alpha}\left(3+\log \frac{2 n-\lambda_{n}}{\lambda_{n}}\right) .
\end{aligned}
$$


For $\alpha=1$, we have

$$
\begin{aligned}
\left\|V_{n}^{a}(\lambda, f)-f\right\|_{\beta} \lesssim & \left(\frac{1+|a|}{1-|a|}\right) \frac{1+\log \lambda_{n}}{\lambda_{n}}\left(1+2 n^{\beta}\right) \\
& +\sup _{0<\delta \leq 1 / n} 2 \delta^{1-\beta}\left(\frac{1+|a|}{1-|a|}\right)\left(3+\log \frac{2 n-\lambda_{n}}{\lambda_{n}}\right) .
\end{aligned}
$$

Therefore we obtain

$$
\left\|f-V_{n}^{a}(\lambda, f)\right\|_{\beta} \lesssim\left\{\begin{array}{cc}
\left(\frac{1+|a|}{1-|a|}\right)^{\alpha} \frac{n^{\beta-\alpha}}{\lambda_{n}^{\alpha}} \log \frac{2 n}{\lambda_{n}}, & \alpha<1 \\
\left(\frac{1+|a|}{1-|a|}\right) n^{\beta-1} \frac{1+\log \lambda_{n}}{\lambda_{n}} \log \frac{2 n}{\lambda_{n}}, & \alpha=1 .
\end{array}\right.
$$

whence (2.2) obviously follows.

\section{References}

[1] DeVore, R. A. and Lorentz, G., Constructive Approximation, Springer-Verlag. New York, 1993.

[2] Holland, A. S. B., A survey of degree of approximation of continuous functions. SIAM Rev. 23 (1981), 344-379.

[3] Huang, C. and Yang L., Approximation by the nonlinear Fourier basis. Sci. China Math. 54 (2011), 1207-1214.

[4] Leindler, L., On summability of Fourier series. Acta Sci. Math. Szeged 29 (1968), 147-162.

[5] Leindler, L., Meir, A. and Totik, V., On approximation of continuous functions in Lipschitz norms. Acta Math. Hung 45 (1985), 441-443.

[6] Prössdorf, S., Zur konvergenz der Fourierreihen hölderstetiger funktionen. Math. Nachr. 69 (1975), 7-14.

[7] Qian, T., Analytic signals and harmonic measures. J. Math. Anal. Appl. 314 (2006), 526-536.

[8] Qian, T. and Chen, Q., Characterization of analytic phase signals. Comput. Math. Appl. 51 (2006), 1471-1482.

[9] Stypinski, Z., On a generalization of the theorem of Prössdorf. Funct. Approx. Comment. Math. 7 (1979), 101-104.

[10] Timan, A. F., Theory of approximation of functions of a real variable, Pergamon Press, 1963.

[11] Zygmund, A., Trigonometric series, Vols. I-II, 2nd edition, Cambridge Univ. Press, London, 1959.

\section{Affiliations}

\section{HATICE ASLAN}

AdDRESS: Firat University, Dept. of Mathematics, 23119, Elazig-Turkey. E-MAIL: haticeaslan2017@gmail.com

ORCID ID: orcid.org/0000-0002-3486-4179

\section{Ali GuVEN}

AdDRESS: Balikesir University, Dept. of Mathematics, 10145, Balikesir-Turkey.

E-MAIL: guvennali@gmail.com

ORCID ID: orcid.org/0000-0001-8878-250X 